\title{
Preliminary Study Using Forward Reaction Control System Jets During Space Shuttle Entry
}

\author{
Carolina Restrepo* and John Valasek ${ }^{\dagger}$ \\ Texas A\&M University, College Station, TX 77843-3141
}

Failure or degradation of the flight control system, or hull damage, can lead to loss of vehicle control during entry. Possible failure scenarios are debris impact and wing damage that could result in a large aerodynamic asymmetry which cannot be trimmed out without additional yaw control. Currently the space shuttle uses aerodynamic control surfaces and Reaction Control System jets to control attitude. The forward jets are used for orbital maneuvering only, while the aft jets are used for yaw control during entry. This paper develops a controller for using the forward reaction control system jets as an additional control during entry, and assesses its value and feasibility during failure situations. Forward-aft jet blending logic is created, and implemented on a simplified model of the space shuttle entry flight control system. The model is validated and verified on the nonlinear, six degree-of-freedom Shuttle Engineering Simulator. A rudimentary human factors study was undertaken using the forward cockpit simulator at Johnson Space Center, to assess flying qualities of the new system and pilot workload. Results presented in the paper show that the combination of forward and aft jets provides useful additional yaw control, in addition to potential fuel savings and the ability to balance the use of the fuel in the forward and aft tanks to meet availability constraints of both forward and aft fuel tanks. Piloted simulation studies indicated that using both sets of jets while flying a damaged space shuttle reduces pilot workload, and makes the vehicle more responsive.

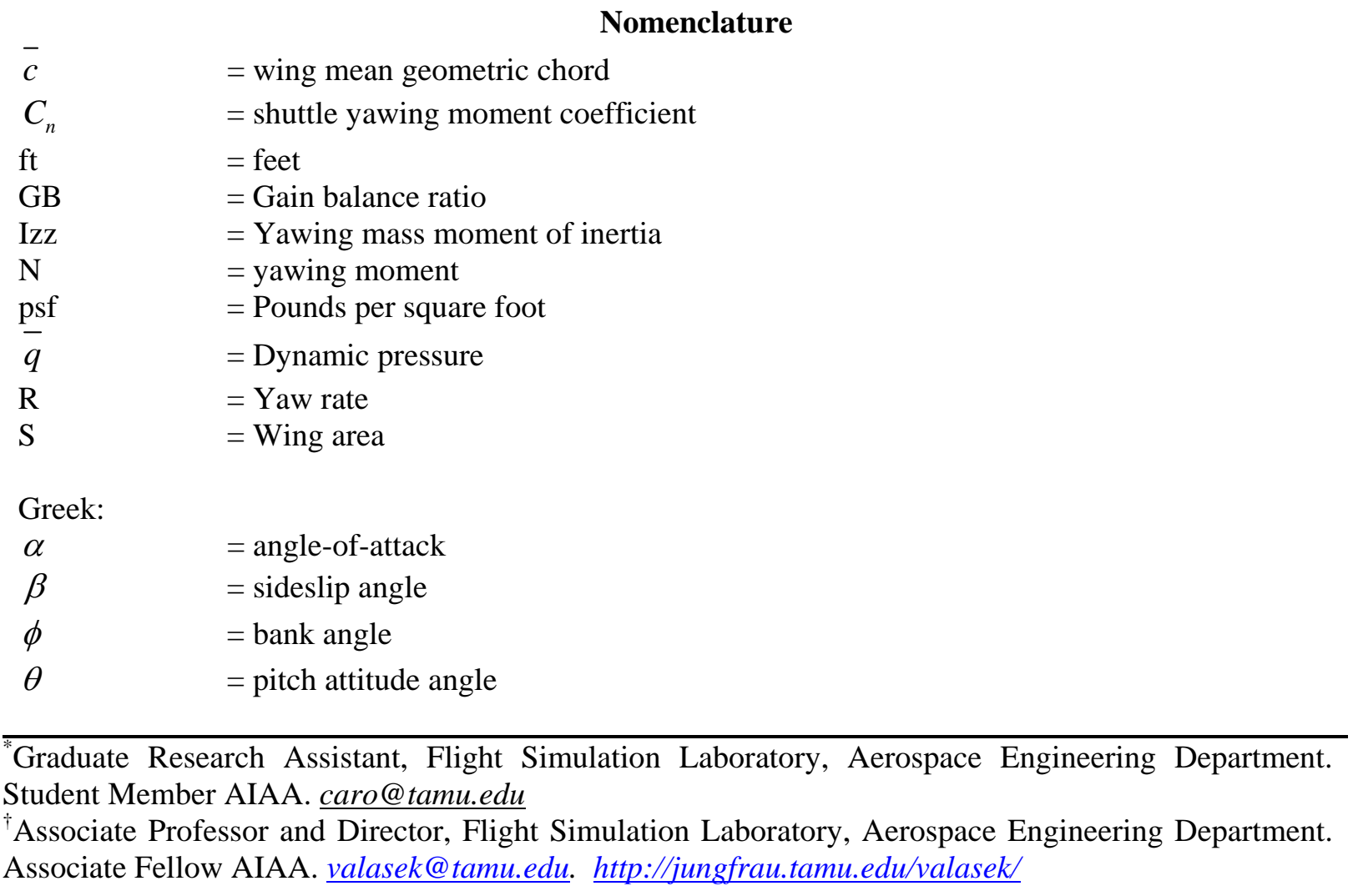




\section{Introduction}

During the entry phase, the shuttle uses both aerodynamic control surfaces and Reaction Control System (RCS) jets to control the vehicle in all axes. In the yaw axis the shuttle has four jets on the aft RCS pods and two on the nose. Currently the vehicle uses only the aft RCS jets for yaw control during entry and the forward jets for orbital maneuvering. The failure or degradation of the aerodynamics or flight control system of the shuttle could result in loss of vehicle control during this phase of flight. Some possible failure scenarios are debris impact or wing damage that can cause large aerodynamic asymmetries that produce large torques. To compensate for these large torques, the vehicle might need to fly at off-nominal attitudes such as large sideslip angles or high angles of attack. In these situations, additional yaw control might be needed. The entry phase is not the only flight condition that could benefit from the use of the forward jets. Another possible scenario is an abort scenario called Glide Return to Launch Site or GRTLS, in which the vehicle flies at very high angles of attack and the control surfaces are not very effective.

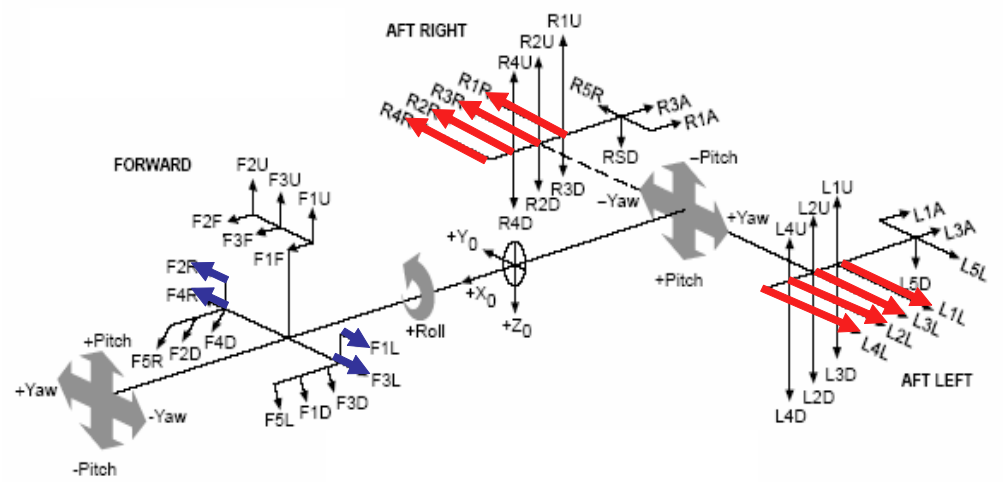

Figure 1. Space Shuttle Reaction Control System

The location of the RCS jets on the shuttle is shown in Figure 1. The aft jets have a moment arm of approximately $40 \mathrm{ft}$. and the forward jets have a moment arm of approximately $60 \mathrm{ft}$. In theory, not including aerodynamic effects, or flow disturbances caused by the firing of the forward jets, firing 2 forward jets can provide the same net torque as firing 3 aft jets. The aerodynamic interaction effects were studied only for low Mach numbers in Ref. 1, but they were not included in the results presented in this paper. In addition to this, there is a requirement that says that a minimum of two aft jets must be fired at the same time. There is no such requirement for the forward jets, so in case a small torque is needed, a single forward jet can be fired instead of two aft jets, which saves fuel.

The shuttle RCS jets are most often used for on orbit maneuvering and attitude control. Although most of the control systems that use RCS jets are in spacecraft, several Earth entry vehicles also use RCS jets during the atmospheric portion of the flight. Typically, only the aft RCS jets are used during entry, due to aerodynamic coupling effects. Previous research has been done on different ways to compensate the torques created by aerodynamic asymmetries or failed control surfaces or RCS jets. Ref. 2 describes a technique to determine whether a failure occurs in the RCS jets or an aerodynamic control surface, by taking specific flight conditions during entry and creating a linear time-invariant system for the lateral dynamics of the orbiter. The nonlinear effects of the jets self-impingement and variations in atmospheric pressure along with other transient effects are ignored, which is similar to the work presented in this paper. A different approach to improve yaw control for the space shuttle during its entry phase considers the use of additional control surfaces parallel to the flow direction and located on the back of the body flap ${ }^{3}$. In addition to the work done with the shuttle entry flight control system, there has been work on other entry vehicles that have RCS jets such as NASA's X-33 and X-38. For example, Ref. 4 describes a control system for the NASA X-38 Crew Return Vehicle, that was designed to detect single or multiple failed RCS jets using only gyro signals. Reference 5 describes research done on flight control systems that are able to detect jammed aerodynamic control surfaces, and can compensate for these failures by using the RCS jets using a model of the X-33 dynamics located in the controller. Additional work was done on reconfiguring flight control systems to handle damaged or jammed control surfaces by using different control allocation techniques, gain scheduling and adaptive control. References 6-9 describe these algorithms, and Ref. 8 describes a fuzzy logic supervised adaptive controller that blends the use of RCS jets and aerodynamic control surfaces for missiles which also use a 
combination of RCS jets and aerodynamic surfaces in a large flight envelope. However, in each of these studies, use of the forward RCS jets was not considered. Spacecraft control systems also try to optimize fuel consumption by designing optimal control systems to manage the RCS thruster firings, an example of this is Ref. 10.

This paper presents a preliminary analysis of the use of both forward and aft jets during the entry phase to provide additional yaw control of the vehicle. The objective of this research is to assess the value and feasibility of using the forward RCS jets of the space shuttle as an additional control during entry, and in particular during failure situations when the integrity of the vehicle or the nominal controller is compromised. Some of the postulated benefits of such a controller are to:

1. Offload the aft RCS

2. Reduce RCS fuel consumption

3. Provide additional torque for a damaged vehicle

4. Provide additional yaw torque to compensate for failed aft jets.

To study the performance of the forward RCS jets, a MATLAB/Simulink simulation model was first developed to study various ways of implementing a forward RCS jet logic. This model was then used to perform a preliminary performance analysis, which formed the basis for implementing the RCS jet logic on the entry flight control system on the nonlinear, high fidelity, six degree-of-freedom Shuttle Engineering Simulator (SES) ${ }^{12}$. Upon validation and verification, the modified code of the SES was ported to the Shuttle forward cockpit simulator at NASA Johnson Space Center. This high fidelity manned real-time simulator was then used to assess the flying qualities of the shuttle when flown with a combination of forward and aft RCS jets.

The paper is organized as follows. Section II develops the analytical model of the Space Shuttle reaction control system model. A MATLAB/Simulink simulation model is developed and described in Section III, and numerical examples are provided. Section IV describes the Space Shuttle Engineering Simulator, and details the modifications made to evaluate the RCS entry controller, including results. Section V describes how the modified code of the SES was run in the forward cockpit simulator at NASA Johnson Space Center, including a brief discussion of the handling qualities of the vehicle flown with a combination of forward and aft RCS jets. This is followed by conclusions and recommendations in Section VI.

\section{Space Shuttle Reaction Control System Model}

The model created for analyzing the effects of the forward RCS jets during the entry phase is a simplified single degree-of-freedom model that includes only the effects of the RCS jets for yaw controls. It does not include any active aerodynamic control surfaces, and the only aerodynamic effect modeled is yawing moment coefficient, $\mathrm{C}_{\mathrm{n}}$, which for this model includes only the effects of weathercock stability, $\mathrm{C}_{\mathrm{n} \beta}$. Ten different sets of constants were used in this study to simulate ten different discrete flight conditions or points during an entry trajectory. A block diagram of the model is shown in Figure 2. Unlike the original SES model, this simplified model does not have an atmosphere model incorporated. Because of this, the first block in Figure 2 has six quantities, bank angle, pitch attitude angle, angle-of-attack, airspeed, dynamic pressure, and Mach number, which describe a flight condition that is kept constant during a simulation run. Another simplification is the decoupling of the yawing and rolling moments. The model includes only the yaw channel block ${ }^{11}$, which is where the forward RCS jet logic was implemented. Inside the yaw channel, the most significant change was rerouting the signal that activates the RCS jets.

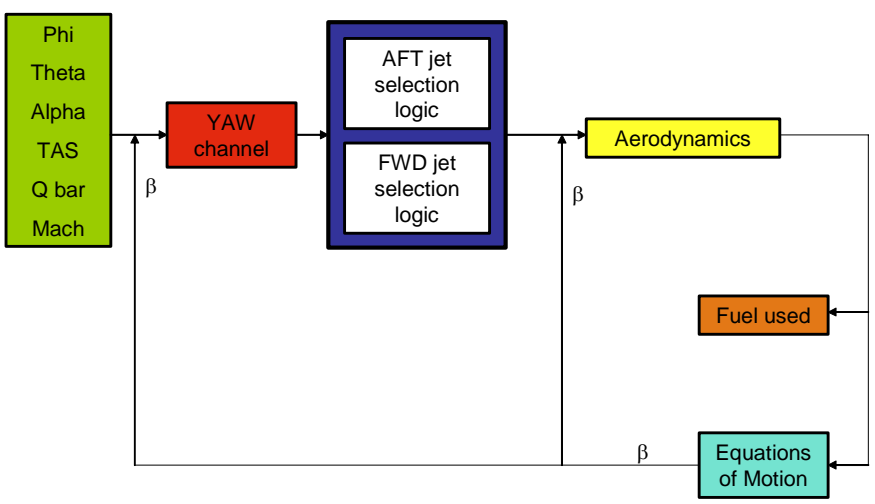

Figure 2. Shuttle RCS Simulink Model

3

American Institute of Aeronautics and Astronautics 
The original model has a signal that is directed to the aft RCS jets, and the modified model has a signal that is divided into two parallel signals: one directed to the aft RCS logic, and another directed to the forward RCS logic. This can be seen in Figure 3. After the signal has been split into the forward and aft jet logic paths, each is multiplied by a different gain. The magnitude of this gain is dependent on the Gain Balance (GB) ratio, which is the value that dictates how much control the forward and aft jets need to provide at a given time. This value is one of three important parameters that were optimized during the simulations.

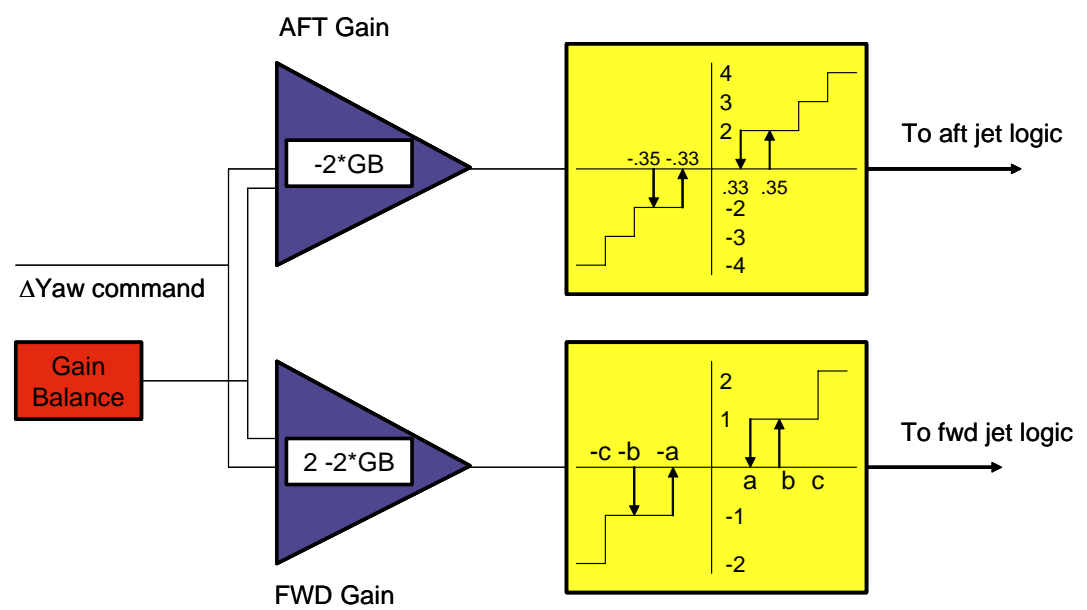

Figure 3. RCS Jet Logic ${ }^{11}$

In addition to the GB ratio modification, separate hysteresis and jet selection logic blocks were added to the model to control the forward jets separately from the aft jets. Having two separate paths for the forward and aft jets had the advantage of allowing the model to use only the aft jets, only the forward jets, or a combination of both. The output of the yaw channel block goes into the jet selection logic blocks for both forward and aft jets, as shown in Figure 2. They determine which of the jets are activated and send a signal to the aerodynamics block, where all the torques are calculated. The aerodynamics block calculates three separate torques: the net forward jet torque, the net aft jet torque, and the torque due to aerodynamic effects. The torque generated by the jets is calculated by multiplying the thrust $(870 \mathrm{lb}$ ) each jet produces during each firing by the moment arm of each jet. In the model, the moment arms were measured from the predicted center of gravity of STS-114, and kept constant during the simulations. The aerodynamic torque is calculated using (1.1), where $C_{n}$ includes the effects of $C_{n_{\beta}}$.

$$
N=\bar{q} S \bar{c} C_{n}
$$

The equations of motion block receives the value for the net torque produced at a given time and it calculates the current yaw rate R using (1.2),

$$
R_{\text {new }}=R_{\text {old }}+\text { RRt }
$$

where $d t=0.04$ seconds and $R=\frac{N}{I_{z z}}$. The current value of sideslip angle is determined using

$$
\beta_{\text {new }}=\beta_{\text {old }}+R d t
$$

The only quantities that are changing during the simulation and are in a feedback loop are the sideslip angle and yaw rate. Another simplification made to the model was the omission of a model of the Wrap Digital Autopilot (DAP). The Wrap DAP, uses a different control logic than the baseline DAP to optimize the use of fuel by combining the use of RCS jets and aerodynamic control surfaces during entry. The model used in this research only has the baseline DAP control logic implemented. 


\section{MATLAB / Simulink Model}

The model described in Section II was converted into a Simulink model, whose yaw channel block model can be seen in Figure 4. The yaw channel was modeled based on the original entry flight control system described in Reference 11. The Simulink model was created primarily to provide a simple platform in which to implement the design of the forward RCS jet logic and test its functionality. The two parallel paths that control the forward and aft RCS jets which depend on the GB ratio are shown in Figure 4, as are the three different sampling rates for different blocks. This multi-rate system has paths sampled at $25 \mathrm{~Hz}, 12.5 \mathrm{~Hz}$, and $6.5 \mathrm{~Hz}$. Since this Simulink model does not have an atmosphere model, ten different points of a nominal entry trajectory were taken, and a set of parameters that describe the flight condition at that point were kept constant during 100 second simulation runs. The set of parameters that describe each flight condition are Mach number, dynamic pressure, angle of attack, pitch angle, bank angle, and airspeed. The parameters for the ten flight conditions used are listed in Table 1. The simulation tests demonstrated that the modified flight control system model worked properly, and that the modifications could be made in the SES.

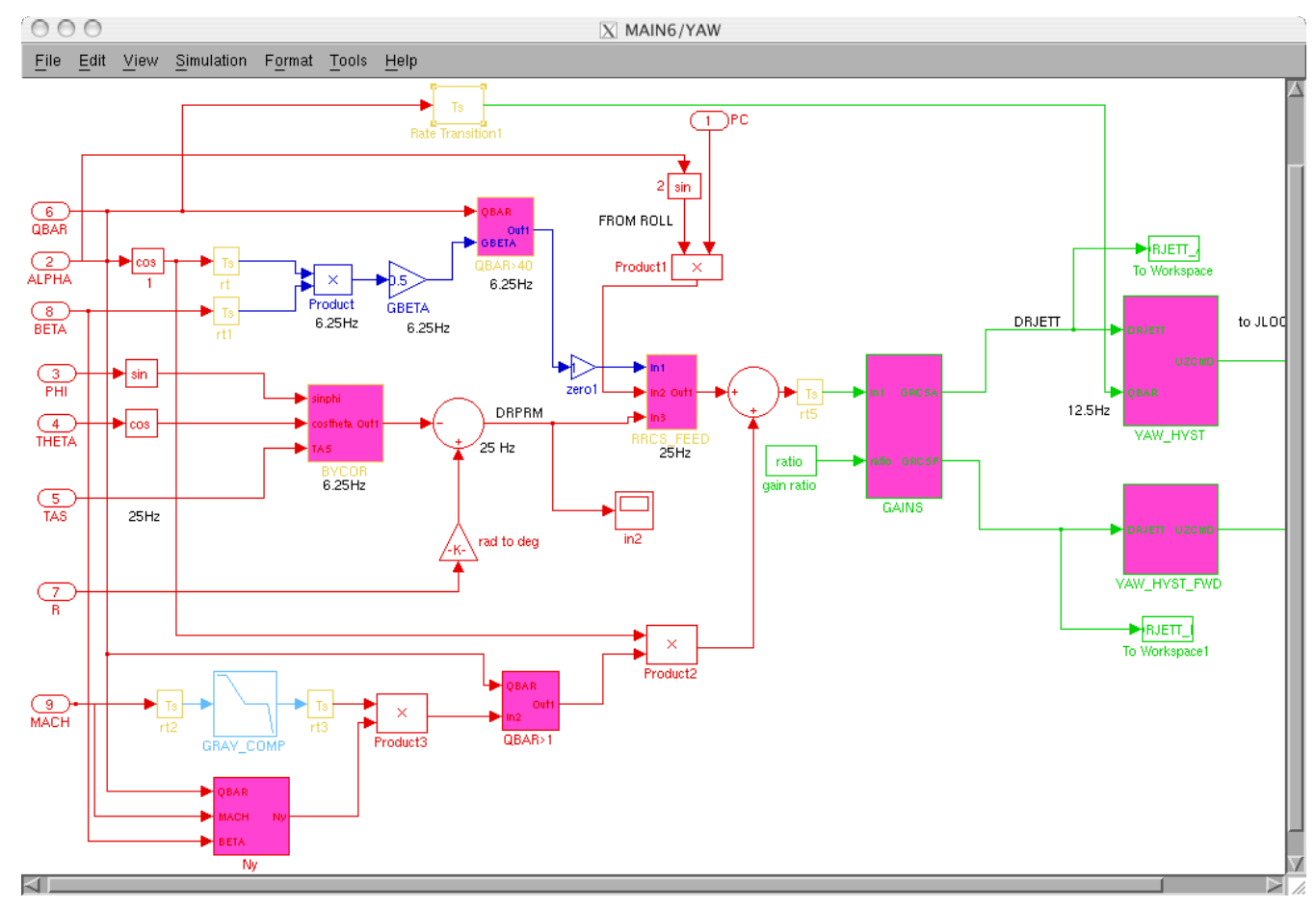

Figure 4. Yaw Channel ${ }^{11}$

The test cases run with the Simulink model provided a range of optimal values for the parameters that were changed during the simulations. These parameters were the following:

- Gain balance ratio - The GB ratio is a value from 0 to 1 . A value of 1 activates the aft RCS jets only. A value of zero activates the forward jets only. Values between zero and one represent a combination of both forward and aft jets. The gain values for the forward and aft jets are directly proportional to the GB value. This is shown in Figure 3.

- Hysteresis dead band $(a, b)$ - The magnitude of $a$ and $b$ dictates when the first jet is turned on and off. If the input signal to the hysteresis block is less than $a$, the jets are off. If the signal is greater than $b$, the jets are turned on. This is shown in Figure 3.

- $2^{\text {nd }}$ jet level $(c)$ - If the input signal is greater than $c$, a second jet is activated. This is shown in Figure 3. 
To determine the optimal values for these parameters, the simulation was run for several flight conditions and the fuel consumption was minimized by changing the parameters. The results obtained were the following. The cases with a GB $=0$ use the least amount of fuel since using only the forward jets provides a larger moment arm and less jet firings are needed to control the shuttle. However, a $\mathrm{GB}=0$ blocks the signal that triggers the aft jets. In case of an emergency, we need to have all jets available instead of only the forward or only the aft. For this reason, all further studies in the Simulink model use GB ratios of 0.25. This can be seen in Figure 5.

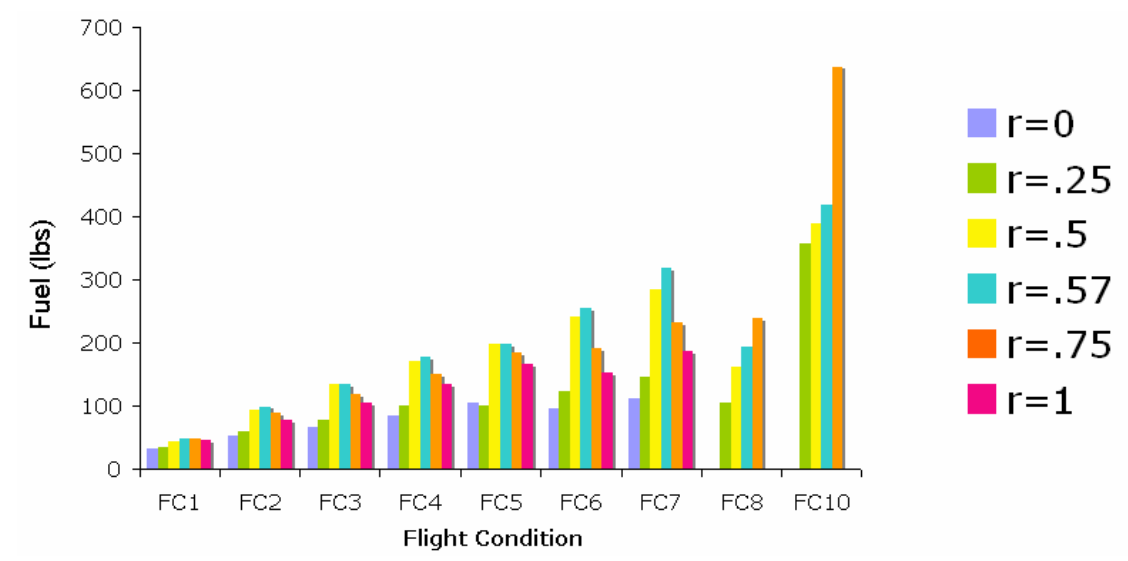

Figure 5. Gain Balance Ratio

The hysteresis dead band size values that help reduce the fuel usage were $0.22-0.24$. Values smaller that these cause limit cycling in certain flight conditions. The results can be seen in Figure 6.
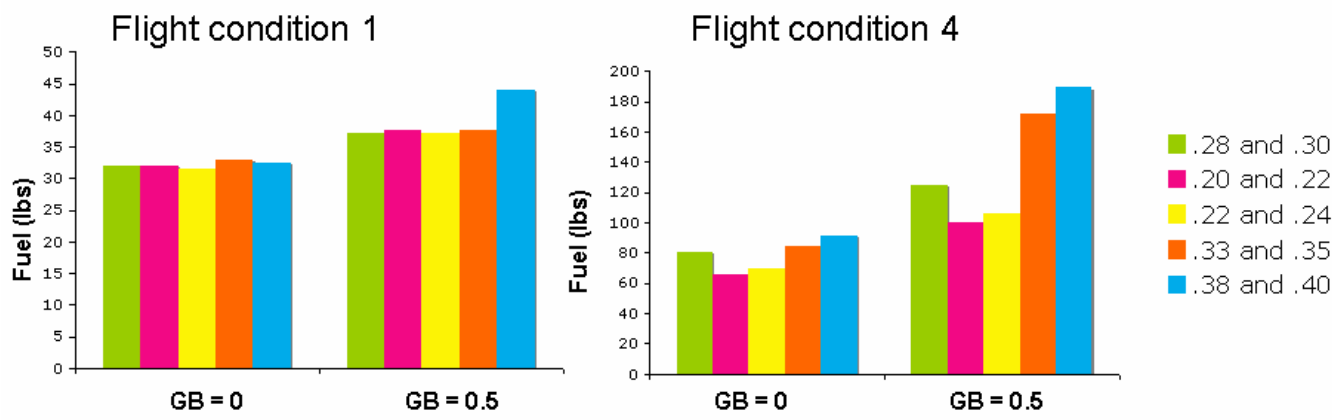

Figure 6. Hysteresis Dead band Size

The second jet level value did not make a big difference in the fuel usage. For this reason, a value of 0.7, similar to the on in the aft logic was chosen. This can be seen in Figure 7.

Flight condition 1

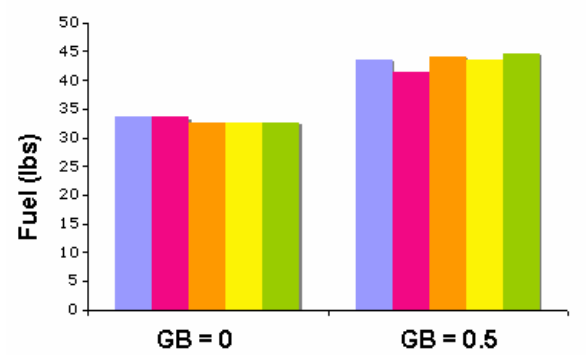

Flight condition 4

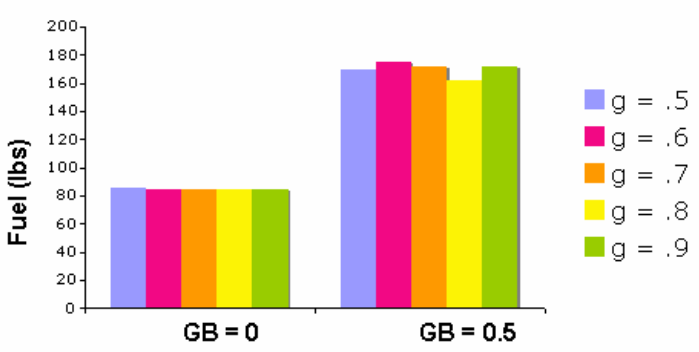

Figure 7. Second Jet Level

American Institute of Aeronautics and Astronautics 
The range of values found for each of the three parameters was used as a starting point for the analysis in the SES. An example of a Simulink model output is described in the following section.

\section{Numerical Examples}

The flight conditions used for these studies are listed in Table 1, and the example presented here is for flight condition 4.

Table 1. STS-114 Flight Conditions for Simulink Model

\begin{tabular}{lcccccccccc}
\hline FC & $\mathbf{1}$ & $\mathbf{2}$ & $\mathbf{3}$ & $\mathbf{4}$ & $\mathbf{5}$ & $\mathbf{6}$ & $\mathbf{7}$ & $\mathbf{8}$ & $\mathbf{9}$ & $\mathbf{1 0}$ \\
\hline \hline Mach & 28.2 & 27.4 & 25 & 22.5 & 20 & 17.5 & 15 & 12.5 & 10 & 7.5 \\
Q-bar & 2 & 16 & 24.9 & 34.7 & 46.5 & 63 & 86 & 107 & 115 & 123 \\
Alpha & 39.6 & 40 & 40 & 40.2 & 40.4 & 40.3 & 39.9 & 40.3 & 39.3 & 34.8 \\
Theta & 38.7 & 15 & 17.5 & 24.9 & 26.7 & 25.6 & 24 & 21.3 & 23 & 18 \\
Phi & 0 & -78 & -69 & -56.2 & -53 & -54.7 & -57.5 & -60.5 & -58 & 58 \\
TAS & 25025 & 24750 & 23400 & 21730 & 19900 & 18000 & 16000 & 13600 & 11000 & 8000 \\
\hline
\end{tabular}

These quantities were kept constant during a 100 second simulation, and the output is shown in Figure 8. The GB ratios used in this example were 0 for forward jets only and 1 for aft jets only. The first plot shows the sideslip angle throughout the simulation. Sideslip angle is initialized at -3 degrees and it is brought back to approximately 0.5 degrees and kept at this value while the jets are firing. The output for the sideslip angle is approximately the same for both cases. The second and third plots show the aft and forward jet firings respectively. It can be seen that the aft jets are fired more often than the forward ones in order to keep the sideslip angle to a minimum. Because of this, the fourth plot shows the fuel used by both forward and aft RCS. Clearly, the forward RCS jets use less fuel.
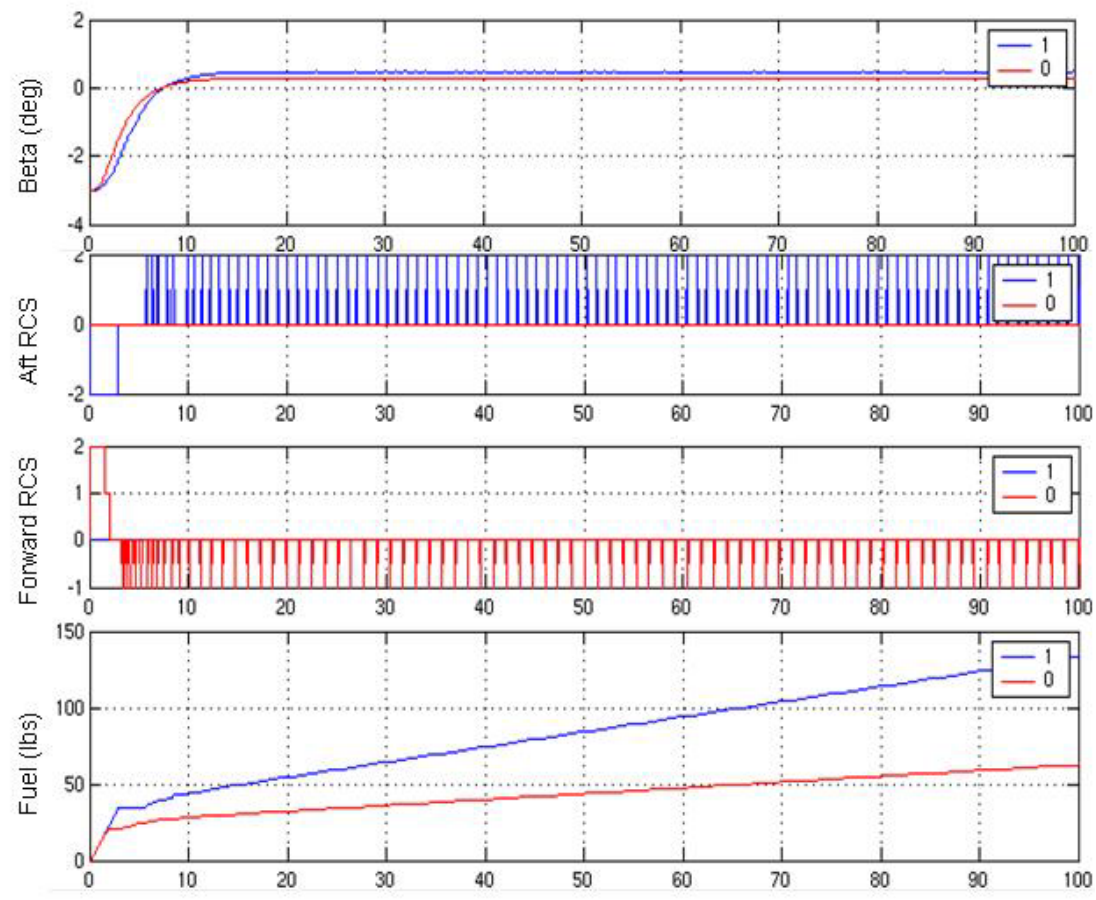

Figure 8. Simulink Model Output Example 


\section{Space Shuttle Engineering Simulator}

The Shuttle Engineering Simulator (SES) is the nonlinear, high fidelity space shuttle simulator at NASA Johnson Space Center. The SES FORTRAN code was modified to implement the same logic for the use of the forward RCS jets that was included in the Simulink model. Three main modules were added: forward hysteresis, forward gain and forward jet logic. The subroutine that calls these routines was modified to call the forward and aft routines to activate the forward and aft jets in proportion to the GB ratio. The starting values for the GB ratio, hysteresis dead band and second jet level were found using the Simulink model. These were used in the different SES runs to verify that the logic was working properly and to compare these values with the Simulink model results. In addition to changing the values of these three parameters, the SES permitted the following conditions to be varied:

- Digital Autopilot (Wrap DAP vs. Baseline DAP)

- Atmosphere conditions ( Calm atmosphere vs. turbulent atmosphere)

- Damaged orbiter vs. nominal orbiter

- Number of failed jets

\section{Numerical Examples}

Figure 9 shows that the total and aft fuel was less for all cases using a combination of forward and aft jets. The results for the fuel usage for the case where the gain balance ration equals 1 (only aft) show that the total fuel usage is higher than all the other cases which use a combination of both forward and aft jets. In addition to reducing the fuel consumption, using a combination of jets can help balance fuel usage between the two tanks. Other potential benefits could be to control the amount of fuel in the tanks. There are cases in which the fuel in either the forward of aft RCS tanks might be limited. Using a combination of forward and aft jets could help control how much fuel from each tank should be used. The simulations did not include a limit in the fuel tanks.

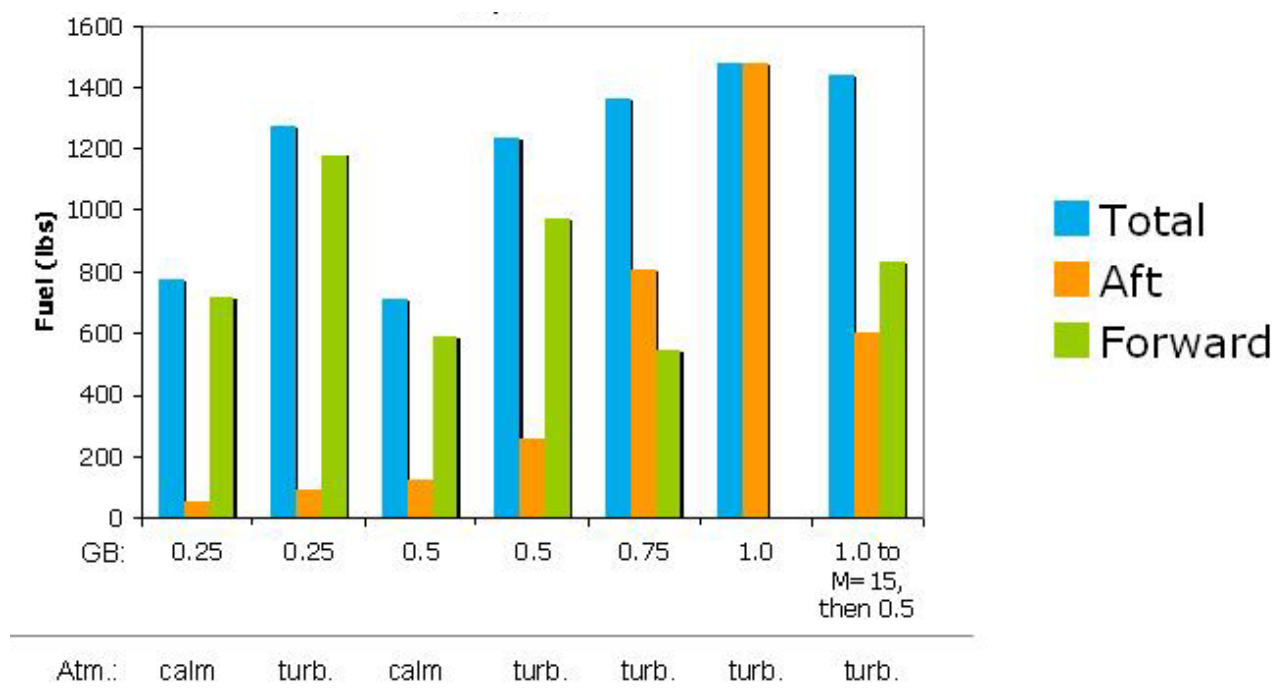

Figure 5. Effects of Atmospheres and Gain Balance Ratio on Fuel Consumption

To evaluate the performance of the modified flight control system, several simulations were run to compare the results between cases that used the Baseline DAP and the Wrap DAP. The results show that both the Wrap DAP and the Base DAP are able to use the forward RCS effectively to augment aft jets. Figure 10 shows the results of the same case ran with both the Baseline DAP (pink) and the Wrap DAP (blue). Comparing some of these cases it was determined that, in general, the forward RCS jets reduce fuel usage. 


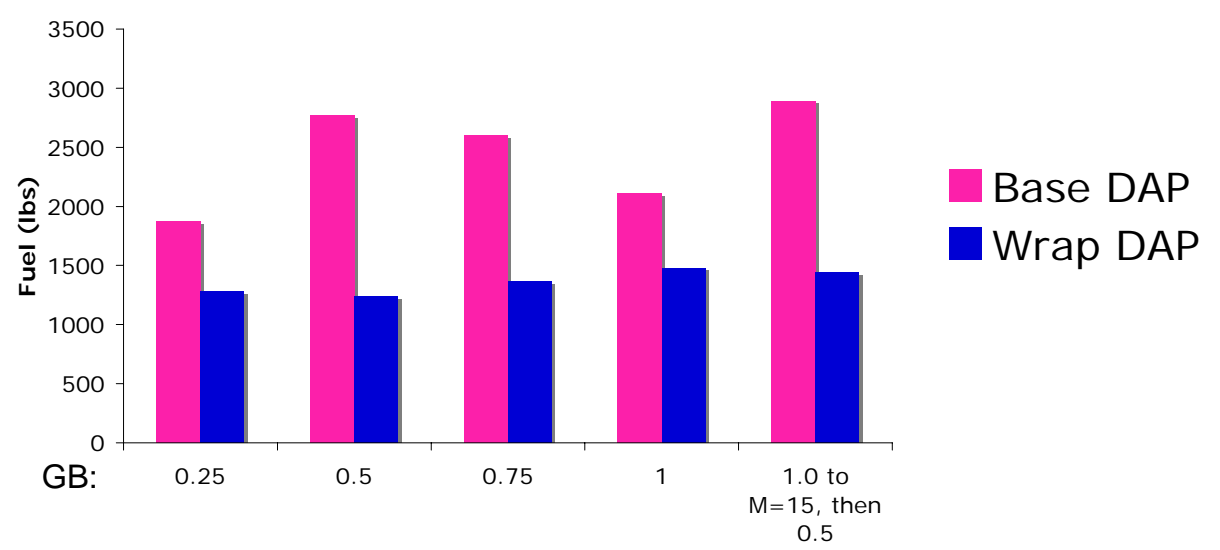

Figure 6. Baseline Digital Autopilot vs. Wrap Digital Autopilot

\section{Forward Cockpit Simulations}

The modified SES code was run in the fixed base forward cockpit simulator at the NASA Johnson Space Center. These runs were used to evaluate the difference in flying qualities of the shuttle during the entry phase. Typically, the vehicle flies with the autopilot until it reaches Mach 1 and then the pilot takes control. For these studies, the vehicle was flown manually during the entire trajectory in order for the pilots to compare the difference in flying qualities for the different cases. All cases ran used the STS-114 shuttle model. Two types of atmosphere models were used. A standard ICAO $1962 \mathrm{calm}$ atmosphere model, and a turbulent atmosphere model. Some of the test cases included simulated failed jets as well as a phantom torque of different magnitudes. The pilots that performed this study were not astronaut pilots.

Aside from the flying qualities, the pilots looked at how difficult it was for the vehicle to "make the runway". After several flights, the comments from two pilots indicated that, in general, the vehicle was easier to maneuver in those cases with a combination of forward and aft jets. The best cases were those with a gain balance ratio of 0.5. In the cases that favored the use of the forward jets, less force on the stick was required but there was a lag in the response of the vehicle. In other words, the shuttle was sluggish and hard to maneuver. In the cases that favored the aft jets, the vehicle was more responsive but the pilot workload was much larger. These results could be improved with astronaut pilots and a blind study that did not tell them which cases they were flying. However, the anecdotal evidence obtained from the cases flown here indicate that using the forward RCS jets can have a positive effect on the perceived handling qualities, and that this can vary with how much emphasis is placed on the use of forward vs. aft jets (GB ratio). The preliminary results presented here suggest that using forward jets can make the vehicle more responsive and less sluggish in its lateral response. Additionally, a balanced mix of forward and aft jets (equal emphasis on fwd and aft, $\mathrm{GB}=0.5$ ) may produce better handling than a 'mix' that simply emphasizes forward jets over aft $(\mathrm{GB}=0.25)$.

\section{Conclusions and Recommendations}

Based on the results presented in this paper, it was determined that the use of forward Reaction Control System jets during the entry phase of a space shuttle flight can provide additional torque to maintain directional control when the aft jets are insufficient. It was also found that the gain balance ratio can be scheduled to balance forward and aft jet activity to save fuel, and to balance fuel usage to stay within availability constraints. The gain balance ratio can also be scheduled to ensure that the forward RCS jets are used only if they are absolutely needed to maintain control of the vehicle, without making any changes to the operation of the aft jets in normal conditions.

Future work will focus on additional validation to create an improved model that includes additional degrees of freedom, as well as more aerodynamic effects. Performance in situations with stuck aero surfaces will also be investigated, and an additional analysis will be done to balance the forward and aft RCS jets with aileron and rudder trim limits. Finally, improved control allocation logic will be developed to track the forward and aft fuel used, in addition to fault detection and isolation logic for failed jets and damage torque. Additionally, a more in depth human factors study will be performed. 


\section{Acknowledgments}

This research was supported by the NASA Langley Research Center, and the NASA Johnson Space Center, under the NASA Co-op Program. The authors gratefully acknowledge this support.

\section{VIII.References}

${ }^{1}$ Covell, Peter F., "Interference Effects of Aft Reaction-Control Yaw Jets on the Aerodynamic Characteristics of a Space Shuttle Orbiter Model”, NASA Technical Memorandum 84645.

${ }^{2}$ Agustin, R.M., Mangoubi, R.S., Hain, R.M., and Adams, N.J., "Robust failure detection for reentry vehicle attitude control systems”, Proceedings of the AIAA Guidance, Navigation, and Control Conference, Boston, MA, August 1998

${ }^{3}$ Scallion, W. I., “Aerodynamic Yaw Controller for the Space Shuttle Orbiter”, Journal of Spacecraft and Rockets, Vol. 2, No. 6, 2005, pp. $1005-1010$

4 Wilson, E., Lages, C., and Mah, R.. “Gyro-based Maximum-likelihood Thruster Fault Detection and Identification,” Proceedings of the 2002 American Control Conference, Anchorage, Alaska, May 2002.

${ }^{5}$ Cotting, M.C., and Burken, J.J. "Reconfigurable Control Design for the Full X-33 Flight Envelope”. NASA Dryden Flight Research Center, NASA/TM-2001-210396, 2001

${ }^{6}$ Burken, J.J., Lu, P., and Wu, Z., “Reconfigurable Flight Control Designs with Application to the X-33 Vehicle”, Proceedings of the Guidance, Navigation, and Control Conference, Portland, OR, August 1999.

${ }^{7}$ Shertzer, R.H., Zimpfer, D.J., Brown, P.D., “Control Allocation for the Next Generation of Entry Vehicles”, Proceedings of the AIAA Guidance, Navigation, and Control Conference and Exhibit, 2002.

${ }^{8}$ Menon, P.K., Iragavarapu, V.R., “Adaptive Techniques for Multiple Actuator Blending”, Proceedings of the AIAA Guidance, Navigation, and Control Conference, Boston, MA, August 1998

${ }^{9}$ Calhoun, P., “An Entry Flight Controls Analysis for a Reusable Launch Vehicle”, Proceedings of the AIAA Aerospace Sciences Meeting, Reno, NV, January 2000

10 Ankersen, Finn, Wu, Shu-Fan, Aleshin, Alexander, Vankov, Alexander, and Volochinov, Vladimir, “Optimization of Spacecraft Thruster Management Function,” Journal of Guidance, Control, and Dynamics, Vol. 28, No. 6, Nov-Dec 2005, pp. 1283-1290.

${ }^{11}$ Space Shuttle Orbiter Operational Level C Functional Subsystem Software Requirements Guidance, Navigation and Control Part C, May 2003

${ }^{12}$ Shuttle Engineering Simulator User's Guide Version 2.0 